\title{
Evaluación de atención selectiva en niños de 7 a 8 años
}

\author{
Evaluation of selective attention in 7-to-8-year-old children
}

1 Diana Catalina Velastegui Hernández

Universidad Técnica de Ambato, Facultad de Ciencias de la Salud. Ambato, Ecuador.

dc.velastegui@uta.edu.ec

2 Rita Elizabeth Velastegui Hernández

Universidad Técnica de Ambato, Facultad de Ciencias de la Salud. Ambato, Ecuador.

rvelastegui1088@uta.edu.ec

3 Jorge Rodrigo Andrade Albán

Universidad Técnica de Ambato, Facultad de Ciencias de la Educación, Carrera de

Psicopedagogía. Ambato, Ecuador.

jr.andrade@uta.edu.ec

4 Rommel Santiago Velastegui Hernández

Universidad Técnica de Ambato, Facultad de Ciencias de la Educación, Carrera de

Psicopedagogía. Ambato, Ecuador.

rs.velastegui@uta.edu.ec

Artículo de Investigación Científica y Tecnológica

Enviado: 24/12/2021

Revisado: 29/12/2021

Aceptado: $12 / 01 / 2022$

Publicado:08/03/2023

DOI: https://doi.org/10.33262/concienciadigital.v6i1.4.2005

Cítese. Velastegui Hernández, D. C., Velastegui Hernández , R. E., Andrade Albán, J. R., \& Velastegui Hernández, R. S. (2023). Evaluación de atención selectiva en niños de 7 a 8 años

ConcienciaDigital,

$6(1.4)$

404-416. https://doi.org/10.33262/concienciadigital.v6i1.4.2005

CONCIENCIA DIGITAL, es una Revista Multidisciplinar, Trimestral, que se publicará en soporte electrónico tiene como misión contribuir a la formación de profesionales competentes con visión humanística y crítica que sean capaces de exponer sus resultados investigativos y científicos en la misma medida que se promueva mediante su intervención cambios positivos en la sociedad. https://concienciadigital.org

La revista es editada por la Editorial Ciencia Digital (Editorial de prestigio registrada en la Cámara Ecuatoriana de Libro con No de Afiliación 663) www.celibro.org.ec 
Palabras

claves:

atención,

atención

selectiva,

stroop, déficit

de atención
Resumen

El estudio propone la evaluación de atención selectiva en una población de estudiantes entre 7 y 8 años, para el proceso de evaluación se ha utilizado prueba stroop, la actividad propuesta es importante para su proceso de aprendizaje debido que están formación y desarrollo neurológico; el estudio plantea una población de 246 educandos de varias unidades educativas del cantón Puyo, Ecuador; obteniendo resultados: 184 (74,79\%) estudiantes puntaje positivo equivalente a "Atención normal", dichos estudiantes han inhibido adecuadamente la respuesta automática que se encuentra en el reactivo; y por otra parte 62 (25.21\%) estudiantes obtienen evaluaciones negativas lo que significa que poseen "déficit de atención" por diferentes factores específicos de cada uno que se analizarán posteriormente.

\section{Keywords:}

selective

attention,

attention,

stroop,

treatment

\begin{abstract}
The study proposes an analysis of selective attention in a population of students between 7 and 8 years old, for the evaluation process the stroop test has been used, the proposed activity is important for their learning process because they are training and neurological development; The study proposes a population of 246 students from various educational units of the Puyo canton, Ecuador; obtaining results: $184(74,79 \%)$ students had a positive score equivalent to "Normal attention", said students have adequately inhibited the automatic response found in the reagent; and on the other hand, 62 $(25,21 \%)$ students obtain negative evaluations, which means that they have "attention deficit" due to different factors specific to each one that will be analyzed later.
\end{abstract}

\section{Introducción}

La atención ha sido estudiada desde diferentes enfoques a lo largo del tiempo, es importante destacar que desde la perspectiva científica de la psicológica clínica y neuropsicológica la atención es el proceso que forma parte de las funciones cognitivas superiores del ser humano (Chen et al., 2018).

No ha logrado homogenizar sus diversas definiciones; se trata sobre un proceso que se activa instantáneamente en todos los seres humanos ante la gran cantidad de estímulos a los que estamos expuestos en forma permanente (Huang et al., 2019). 
El proceso atencional se evidencia al limitar o filtrar la cantidad de información que recibimos y permitir que solo los estímulos o estímulos más relevantes a través de los órganos sensoriales ingresen al sistema nervioso central (Marotta et al., 2018).

Está directamente relacionado con las funciones ejecutivas ubicadas a nivel de la corteza prefrontal del lóbulo frontal. Dentro de las funciones cognitivas superiores, se pueden citar varias de ellas como la memoria, el pensamiento, la atención, entre otras. La atención es considerada como un proceso complejo donde el ser humano interactúa con el entorno obteniendo estímulos, puede ser de diferentes tipos, entre estos podemos referirnos a la atención selectiva cuyas características destacan en poner la atención en estímulos sobresalientes (externos) previniendo otros menos notables. estímulos generadores de distractores (Motesa et al., 2018).

Las teorías evolutivas y las funciones cognitivas evolucionan según la edad del individuo de manera que a medida que pasan los años mejoran notablemente funciones superiores como la atención y sus tipos (Guillamón, et al., 2021).

Los procesos cognitivos superiores son diferentes según el estudio que se realice, no se puede garantizar que la evaluación sea generalizada, en edades escolares se puede demostrar que los procesos atencionales son diversos, los niños entre 9 y 11 años presentan procesos atencionales que en algunos casos tienen diferentes tipos de afectación propios de su edad, teniendo en cuenta que esta etapa está llena de estímulos, se toma como un "proceso de formación" que beneficia a los diferentes tipos de atención a la perfección (Carpio, 2020).

La atención selectiva tiene características inmersas de control inhibitorio y velocidad de procesamiento, estas particularidades son estimulables, es decir, se pueden entrenar con el tiempo para que su función muestre mejoras significativas. La inhibición y la velocidad de procesamiento son solo dos de los mecanismos cognitivos importantes en juego en las actividades cognitivas humanas (Introzzi et al., 2019).

Si se considera la perspectiva del marco clínico psicopatológico entonces la atención y las funciones superiores pueden sufrir alteraciones a lo largo de la vida. La mayoría de las afectaciones se evidencian precisamente en la edad escolar ya que es en esta etapa de la vida donde el ser humano pone en juego sus habilidades atencionales para poder abstraer información externa que le permita adquirir nuevos conocimientos (Wray et al., 2017).

Gran parte de los problemas de atención de los niños están relacionados con los procesos de aprendizaje a los que son sometidos dentro del sistema escolar (Lagos et al., 2019).

Al enfocar la teoría en el cerebro, es necesario enfatizar que se concibe como una estructura integral ya que trae como un todo, sus diferentes córtex y diversas divisiones 
participan en cada uno de los procesos que realiza el sistema, aunque también es cierto que según la función que se realice, unas áreas tomarán más protagonismo que otras (Berardi et al., 2021).

Dentro de las bases neuropsicológicas de la atención, cabe señalar que esta función superior y sus diferentes tipos dependen del funcionamiento de varias estructuras cerebrales importantes como es el sistema activador reticular ascendente, también conocido como SARA, este sistema se define de forma elemental, el nivel más básico para iniciar el proceso atencional, viéndolo desde la perspectiva de que para poder prestar atención a un estímulo se requiere que todo nuestro sistema esté listo para percibir. El tono y la vigilia, que son aspectos indispensables en los procesos cognitivos superiores, incluida la atención, permiten que el sistema - el cuerpo - mantenga un estado de alerta, lo que hace que sea receptivo a la estimulación y preparación, lo que le permite dar una respuesta correcta (Fritze et al., 2021).

Los colículos superiores ubicados en el mesencéfalo participan en los cambios de estímulos atencionales relacionados con los estímulos visuales, los colículos superiores favorecen el foco de atención a nuevos lugares u objetos controlando los movimientos oculares encargados de llevar los estímulos periféricos al área visual (Evensberget et al., 2011).

Los ganglios basales son una formación de núcleos subcorticales que rodean al tálamo, el más prominente es el caudado seguido del putamen y el globo pálido ubicado entre el tálamo y la ínsula. Su función es motora, se activan en la selección, percepción y respuestas (Nee et al., 2007).

El núcleo pulvinar del tálamo recibe casi todos los estímulos sensoriales que ingresan al sistema, desde los cuales se envían a diferentes partes del cerebro (Passingham et al., 2000).

La corteza cingulada integra toda esta información con la emoción y ayuda a seleccionar una respuesta adecuada (Lima Giacobbo et al., 2019)

Debido a estos importantes aportes de diferentes investigaciones y a la falta de estudios dirigidos puramente a la evidencia infantil, se propuso analizar la atención selectiva en bebés entre 9 y 11 años evaluando la velocidad de procesamiento e inhibición al desempeño en una tarea. Para ello, se administró una prueba de Stroop para evaluar la capacidad de clasificar información del entorno y reaccionar selectivamente a ella.

\section{Metodología}

La investigación es necesaria porque existen escasos trabajos científicos sobre este tema, más aún cuando se maneja un excelente número de evaluaciones de atención a través de 
la prueba de Stroop, considerando que los datos obtenidos de la población de estudio se encuentran en la etapa escolar donde se destacan los problemas de aprendizaje. y generalmente están vinculados a este tipo de dificultades.

Finalmente, al identificar las alteraciones atencionales de los estudiantes, a partir del uso del instrumento psicológico, será posible diagnosticar procesos terapéuticos que refuercen las deficiencias encontradas en estudiantes de 7 a 8 años.

\section{Diseño y población}

En este estudio participaron 246 estudiantes de siete y ocho años. No se calculó la muestra y por lo tanto se aplicó la prueba a toda la población. Los participantes pertenecían a varias escuelas de Puyo, Ecuador. La prueba se aplicó durante el primer parcial del segundo semestre del curso escolar 2020.

Se consideraron los siguientes criterios de inclusión: (i) participación voluntaria en el estudio; (ii) pertenecer al grupo poblacional de 7 a 8 años; (iii) área de operación - en Puyo; y (iv) los estudiantes no deben presentar necesidades especiales.

\section{Instrumento de recolección de información}

Se utilizó la prueba de color y palabra Stroop; la evaluación de la prueba se realizó de manera individual, el tiempo de duración fue de aproximadamente 300 segundos para cada alumno; la evaluación contiene tres hojas de papel tamaño A4, cada una de las cuales contiene 100 ítems distribuidos en cinco columnas de 20 ítems cada una.

Las diapositivas se presentan de la siguiente manera: (i) Palabras (P), que consisten en las palabras ROJO, VERDE y AZUL ordenadas al azar; todas las palabras estaban impresas en tinta negra. No está permitido imprimir dos palabras repetidas en la misma columna. (ii) Colores (C), que constan de cuatro $\mathrm{X}$ "XXXX" impresas en azul, verde y rojo. No se permite imprimir el mismo color dos veces en la misma columna y el orden de impresión es diferente al de la primera hoja; (iii) Word-Color (PC) que contiene palabras de la primera hoja en los colores de la segunda hoja. El color y la palabra no deben coincidir en ningún caso con el color de la tinta; por lo tanto, el alumno solo debe mencionar el color de la tinta.

Con los resultados obtenidos, debemos aplicar escalas para comparar la equivalencia; sin embargo, en este caso, obtenemos el resultado bruto.

\section{Evaluación individual}

Una vez que el evaluador ha verificado la disponibilidad de los recursos necesarios para la aplicación de la prueba, se deben tomar en consideración las siguientes instrucciones: (i) presentar las hojas de P, C y PC al estudiante evaluado; (ii) las hojas se presentan frente 
al alumno evaluado en una superficie plana; (iii) el alumno evaluado no podrá girar la hoja más de $45^{\circ}$ a derecha o izquierda, así mismo no se le permitirá levantar o separar la hoja de la superficie donde fue colocada; (iv) la tercera página debe estar ubicada en la misma posición que la segunda página; y, (v) no se permite cubrir ninguna hoja.

\section{Puntuación T}

Una vez culminado el proceso de evaluación individual, se ha procedido a realizar la Puntuación T la cual consiste en los siguientes pasos:

(a) P: es el resultado del número de palabras leídas correctamente en la primera lámina; $\mathrm{C}$ : es el resultado del número de elementos nombrados correctamente en la segunda lámina de los colores; $\mathrm{y}, \mathrm{PC}$ es el resultado de elementos nombrados correctamente en la tercera lámina. Los errores cometidos no puntúan.

Tabla 1

Baremos prueba Stroop

\begin{tabular}{ccccc}
\hline$P$ & $C$ & $P C$ & Interferencia & Puntuación $T$ \\
\hline 168 & 125 & 75 & 30 & 80 \\
164 & 122 & 73 & 28 & 78 \\
160 & 119 & 71 & 26 & 76 \\
156 & 116 & 69 & 24 & 74 \\
152 & 113 & 67 & 22 & 72 \\
148 & 110 & 65 & 20 & 60 \\
144 & 107 & 63 & 18 & 66 \\
140 & 104 & 61 & 16 & 64 \\
136 & 101 & 59 & 14 & 62 \\
132 & 98 & 57 & 12 & 60 \\
128 & 95 & 55 & 10 & 58 \\
124 & 92 & 53 & 8 & 56 \\
120 & 89 & 51 & 6 & 54 \\
116 & 86 & 49 & 4 & 50 \\
112 & 83 & 47 & 2 & 48 \\
108 & 80 & 45 & 0 & 46 \\
104 & 77 & 43 & -2 & 44 \\
100 & 74 & 41 & -4 & 42 \\
96 & 71 & 39 & -6 & 40 \\
92 & 68 & 37 & -8 & 38 \\
88 & 65 & 35 & -10 & 36 \\
84 & 62 & 33 & -12 & 34 \\
80 & 59 & 31 & -14 & 32 \\
76 & 56 & 29 & -16 & 30 \\
72 & 53 & 27 & -18 &
\end{tabular}


Tabla 1

Baremos prueba Stroop (continuación)

\begin{tabular}{ccccc}
\hline $\boldsymbol{P}$ & $\boldsymbol{C}$ & $\boldsymbol{P C}$ & Interferencia & Puntuación $\boldsymbol{T}$ \\
\hline 64 & 47 & 23 & -22 & 28 \\
60 & 44 & 21 & -24 & 26 \\
56 & 41 & 19 & -26 & 24 \\
52 & 38 & 17 & -28 & 22 \\
48 & 35 & 15 & -30 & 20 \\
\hline
\end{tabular}

Fuente: Baremos prueba Stroop

(b) Para nuestra población de estudio (estudiantes de 7 a 8 años) se considera el puntaje en bruto es decir el número de aciertos leídos y nombrados.

(c)Luego se procede a calcular PC' (PC estimada) de acuerdo con la siguiente fórmula:

$\mathrm{PC}^{\wedge}=(\mathrm{P} * \mathrm{C}) /(\mathrm{P}+\mathrm{C})$

(d)Después se calcula la Interferencia aplicando la siguiente ecuación:

Interferencia $=\mathrm{PC}^{\mathrm{P}} \mathrm{PC}^{\prime}$

(e)El valor de interferencia servirá para realizar una relación con los baremos y encontrar la Puntuación T, los baremos de transformación se encuentran en la Tabla 1.

\section{Consolidación y análisis de la información}

Finalmente, una vez realizada la evaluación de 135 estudiantes y obtenidos los valores de P, C, PA, se consolidó la información en una hoja de Excel para obtener los valores de PA ', Interferencia y T-score. Posteriormente, se utilizó el software gratuito RStudio para descartar errores en los valores obtenidos.

\section{Aspectos éticos}

El consentimiento informado se aplicó individualmente a los padres o representantes de cada estudiante. Durante la socialización se dio a conocer la finalidad del estudio, el manejo seguro y confidencial de los datos, así como el uso con fines académicos.

\section{Resultados}

Una vez aplicada la prueba de colores y palabras Stroop, se obtienen los siguientes resultados: (i) interferencia y (ii) Puntuación T.

Resultados de la interferencia 
La evaluación de la interferencia según esta prueba recomienda que valores inferiores a "0" equivalen a "Déficit de atención" y superiores a "1" equivalen a "Atención normal"; sin embargo, debido a la experiencia en casos clínicos en estudiantes de 7 a 8 años, se consideró que la evaluación de "Interferencia menor a 10" serán casos para análisis e intervención individual.

Por tanto, una vez aplicada la prueba, los resultados obtenidos en la población de estudio se encuentran en el rango de -14 a 18; las evaluaciones negativas, considerando el rango propuesto, indican que en los niños el desarrollo evolutivo no existe el mismo nivel de selectividad, debido a que tienen dificultad para interferir con los estímulos, están expuestos a ciertos estímulos externos, lo que equivale a "Déficit de atención" como se muestra en la Figura 1.

\section{Figura 1}

Resultado de interferencia, a través de RStudio

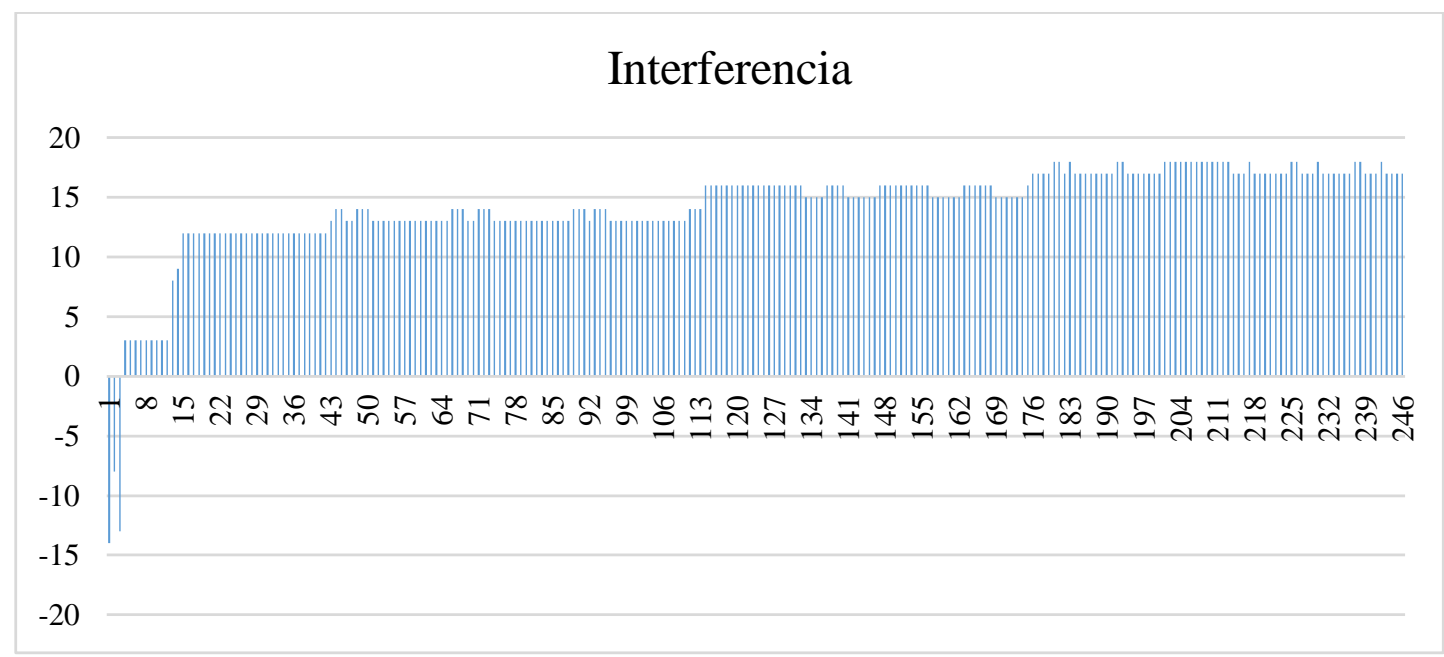

Nota: Evaluación de 246 estudiantes del Cantón Puyo

Resultados de la puntuación T

Una vez obtenidos los valores de interferencia, se aplicó la escala indicada en la Tabla 1, y se obtuvieron valores que oscilan entre 36 y 68.

Por tanto, 127 estudiantes (94\%) con puntuación positiva equivalente a "Atención normal" han inhibido adecuadamente la respuesta automática encontrada en el reactivo; Por otro lado, las evaluaciones negativas se han considerado a partir del valor bruto de la interferencia menor o igual a (interferencia $=10$ ) resultando en 8 estudiantes $(6 \%)$ presentando una puntuación negativa, lo que indica que presumiblemente han inhibido inadecuadamente las respuestas, equivalente a un "Déficit de atención". 


\section{DDigital}

\section{Figura 2}

Resultado de Puntuación T en RStudio

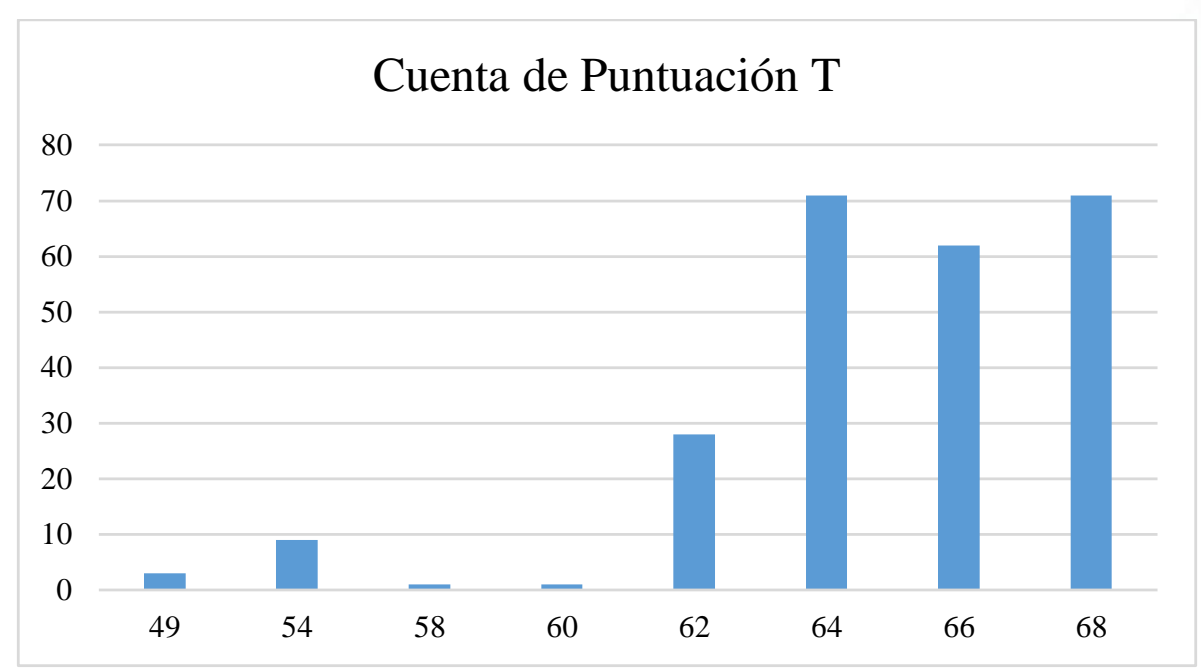

Nota: Evaluación de 246 estudiantes del Cantón Puyo

Si la puntuación es positiva, el individuo ha inhibido adecuadamente la respuesta automática y, si es negativa, presumiblemente la ha inhibido inadecuadamente (siempre considerando que la población general oscila aproximadamente entre -10 y 10).

Además, como resultado final, la Figura 3 muestra una comparación entre la interferencia y la puntuación $\mathrm{T}$.

\section{Figura 3}

Resultado de Puntuación T en RStudio

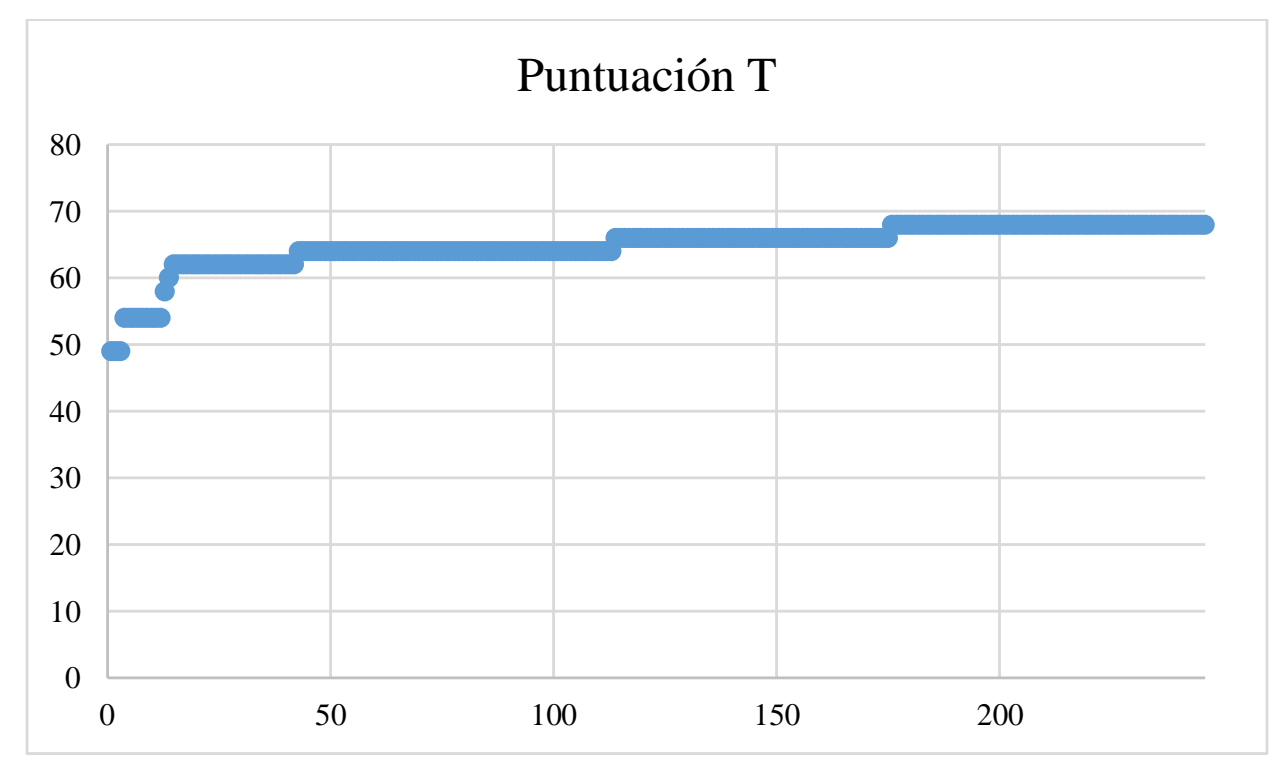

Nota: Evaluación de 246 estudiantes del Cantón Puyo 


\section{Conclusiones}

- De la población de 246 estudiantes entre 7 y 8 años, que participaron en el proyecto, se han obtenido las siguientes evaluaciones: puntajes positivos con base en la experiencia clínica del desarrollo cerebral, este porcentaje obtenido debe analizarse ampliando el nivel de puntuación de interferencia negativa de manera que se haya obtenido el $74,79 \%$ de puntuaciones positivas lo que equivale a 184 estudiantes con una puntuación positiva equivalente a "Atención Normal". "lo que significa que han inhibido adecuadamente la respuesta automática encontrada en el reactivo.

- Las evaluaciones negativas se han considerado a partir del valor bruto de interferencia menor o igual a (interferencia $=10$ ) resultando en 62 estudiantes que presentan un puntaje negativo, lo que indica que presumiblemente, han inhibido peor de lo esperado al momento de obteniendo los puntajes de la evaluación y tiene una equivalencia de "Déficit de atención" para diferentes factores que posteriormente serán analizados.

- El desarrollo de las funciones ejecutivas en general y la atención selectiva, en particular, es irregular entre los niños del mismo grupo de edad. Esto es de particular importancia a la hora de planificar programas educativos y abordar las dificultades de aprendizaje que los estudiantes pueden encontrar cuando se enfrentan a contenidos educativos.

- Se puede observar que los niños entre 7 y 8 años que fueron sometidos a la evaluación del instrumento muestran resultados diferentes, algunos por debajo de la norma, lo que nos permite mostrar que existen algunas posibles deficiencias en la atención selectiva ya que aún no lo han hecho. es lo suficientemente maduro para poder ignorar los estímulos que distraen.

- Los puntajes iniciales de los niños evaluados tienen niveles estándar, según figura 3, y las interferencias comienzan a notarse desde la mitad de la ilustración, por lo que se puede concluir que los niños que terminaron la aplicación primero lo hicieron correctamente denotando que su control inhibitorio El proceso y la velocidad de procesamiento están de acuerdo con su edad y, por lo tanto, su etapa evolutiva.

- Los niños que se encuentran a partir de la mitad de la figura 3, presentan mayor cantidad de interferencias y su tiempo de resolución de la prueba fue mayor, por lo que es evidente que su proceso de atención selectiva aún no ha logrado concretar sus procesos de control y procesamiento inhibitorio La velocidad no significa que estén equivocados, sino que estos procesos posiblemente estén un poco "retrasados" con respecto a su edad y etapa evolutiva. 


\section{Agradecimientos}

Los autores agradecen a la Universidad Técnica de Ambato (UTA) y a la Dirección de Investigación y Desarrollo (DIDE) por su apoyo brindado para la ejecución exitosa de este trabajo a través del proyecto de investigación titulado "Potenciación de funciones ejecutivas a través de un programa de entrenamiento cerebral y aprendizaje mediado". A la Universidad Complutense de Madrid por su colaboración.

Un homenaje póstumo al PhD Paúl Santiago Pullas Tapia quien fue coordinador inicial del proyecto de investigación e impulsó con su liderazgo inigualable la creación y desarrollo de actividades educativas y científicas en favor de la juventud ecuatoriana, para que desde el cielo nos guíe en el camino de la verdad y de la honestidad.

\section{Referencias bibliográficas}

Berardi, A., Panuccio, F., Pilli, L., Tofani, M., Valente, D. \& Galeoto, G. (2021). Evaluation instruments for executive functions in children and adolescents: a systematic review. Expert Review of Pharmacoeconomics and Outcomes Research, 21(5), 885-896. https://doi.org/10.1080/14737167.2021.1908889

Carpio, B. (2020). desarrollo de la atención selectiva a través del juego en estudiantes de educación superior. Comunicación, 131-141.

Chen, M., Herrera, F. \& Hwang, K. (2018). Cognitive Computing: Architecture, Technologies, and Intelligent Applications. IEEE Access, 19774 - 19783. doi:10.1109/ACCESS.2018.2791469.

Evensberget, D., Brunskill, C., Johnson, C., McCrum, M., Osborne, J. R., Weiss, B. M., Eriksson, K. \& Ewald, R. (2011). An interdisciplinary approach to human-robotic cooperation in Mars exploration. 62nd International Astronautical Congress 2011, IAC 2011, 2, 1661-1670. https://doi.org/10.2139/ssrn.2120254

Fritze, S., Harneit, A., Waddington, J. L., Kubera, K. M., Schmitgen, M. M., Otte, M. L., Geiger, L. S., Tost, H., Meyer-Lindenberg, A., Wolf, R. C. \& Hirjak, D. (2021). Structural alterations in brainstem, basal ganglia and thalamus associated with parkinsonism in schizophrenia spectrum disorders. European Archives of Psychiatry and Clinical Neuroscience, 271(8), 1455-1464. https://doi.org/10.1007/S00406-02101270-Y

Guillamón, A., García, E., \& Martínez, H. (2021). Ejercicio físico aeróbico y atención selectiva en escolares de educación primaria. Retos, 421-428. 
Huang, L., Wang, W., Chen, J. \& Wei, X.-Y. (2019). Attention on Attention for Image Captioning. Proceedings of the IEEE/CVF International Conference on Computer Vision, 4634-4643.

Introzzi, I., Aydmune, Y., Zamora, E., \& Vernucci, S. (2019). Mecanismos de desarrollo de la atención selectiva en población infantil. CES Psico, 105-118.

Lagos, R., Pizarro, D., \& Fuentes, G. (2019). Programa de desarrollo cognitivo y motor para atención selectiva y sostenida de niños con TDAH. Educación, 411-425.

Lima Giacobbo, B., Doorduin, J., Klein, H. C., Dierckx, R. A. J. O., Bromberg, E., \& de Vries, E. F. J. (2019). Brain-Derived Neurotrophic Factor in Brain Disorders: Focus on Neuroinflammation. Molecular Neurobiology, 56(5), 3295-3312. https://doi.org/10.1007/S12035-018-1283-6

Marotta, A., Caballero, R., Lupiáñez, R., \& Arrows, J. (2018). The arrows don't look at you: qualitatively different attention mechanisms activated by the look and the arrows. Qualitatively different attentional mechanisms triggered by gaze and arrows. Psychon Bull Rev 25, 2254-2259.

Motesa, M. A., Yezhuvath, U. S., Aslan, S., Spence, J. S., Rypma, B., \& Chapma, S. B. (2018). Higher-order cognitive training effects on processing speed-related neural activity: a randomized trial. Neurobiology of Aging, 72-81.

Nee, D. E., Wager, T. D., \& Jonides, J. (2007). Interference resolution: Insights from a meta-analysis of neuroimaging tasks. Cognitive, Affective and Behavioral Neuroscience, 7(1), 1-17. https://doi.org/10.3758/CABN.7.1.1

Passingham, R. E., Toni, I., \& Rushworth, M. F. S. (2000). Specialization within the prefrontal cortex: the ventral prefrontal cortex and associative learning. Executive Control and the Frontal Lobe: Current Issues, 103-113. https://doi.org/10.1007/9783-642-59794-7_12

Wray, A. H., Stevens, C., Pakulak, E., Isbell, E., Bel, T., \& Neville, H. (2017). Development of selective attention in preschool-age children from lower socioeconomic status backgrounds. Developmental Cognitive Neuroscience, 101111.

\section{Ciencia LDigital


El artículo que se publica es de exclusiva responsabilidad de los autores y no necesariamente reflejan el pensamiento de la Revista Conciencia Digital.

\section{Ciencia \\ LDigital}

El artículo queda en propiedad de la revista y, por tanto, su publicación parcial y/o total en otro medio tiene que ser autorizado por el director de la Revista Conciencia Digital.
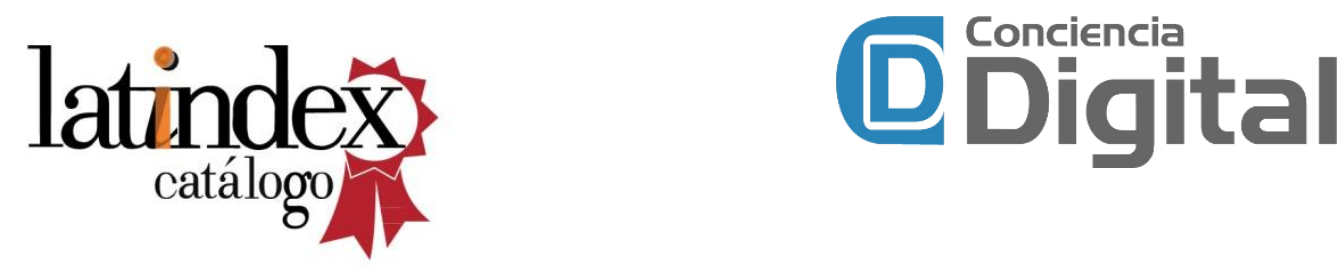

Indexaciones

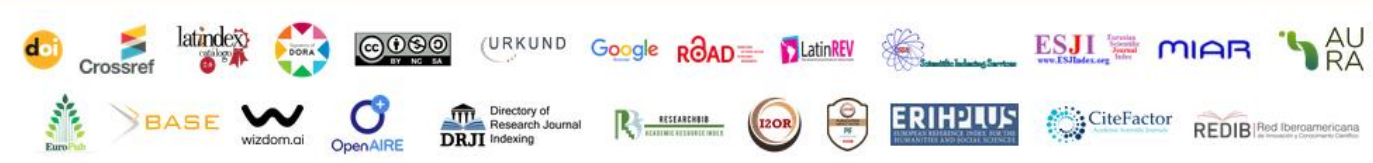

